\title{
Reconsidering the Inverse Gambler's Fallacy Charge Against the Fine-Tuning Argument for the Multiverse
}

\author{
Simon Friederich ${ }^{1,2}$ (D) \\ Published online: 22 June 2018 \\ (C) The Author(s) 2018
}

\begin{abstract}
Does the claimed fine-tuning of the constants of nature for life give reason to think that there are many other universes in which the constants have different values (a "multiverse")? Or does the inference from fine-tuning to a multiverse commit what Hacking calls the inverse gambler's fallacy? The present paper considers two fine-tuning problems that seem promising to consider because they are in many respects analogous to the problem of the fine-tuned constants. Reasoning that parallels the inference from fine-tuning to a multiverse seems prima facie adequate in these problems. However, it turns out that in both cases there are independent empirical reasons to believe the hypotheses that are analogous to the multiverse hypothesis. In the absence of such evidence, it would be coherent to raise the inverse gambler's fallacy charge against the inference from fine-tuning to these multiverse-type hypotheses. In response to this finding, I suggest taking the possibility seriously that established standards of rationality may not allow us to decide whether the inference from fine-tuning to a multiverse is fallacious or not. The paper concludes by sceptically assessing the prospects for obtaining independent empirical evidence for concrete multiverse theories.
\end{abstract}

Keywords Fine-tuning · Multiverse · Anthropic reasoning · Inverse gambler's fallacy

\section{Introduction}

Are there other universes, some perhaps radically different from our own? Some physicists and philosophers are attracted to this multiverse idea because they regard it as offering a promising response to the puzzle that many constants of nature appear to be

Simon Friederich

email@simonfriederich.eu; s.m.friederich@ rug.nl

1 University of Groningen, University College Groningen, Hoendiepskade 23/24, 9718, BG, Groningen, The Netherlands

2 University of Groningen, Faculty of Philosophy, Oude Boteringestraat 52, 9712, GL, Groningen, The Netherlands 
fine-tuned for life: had they been even slightly different, life as we know it could not have existed. ${ }^{1}$

Many regard the apparent fine-tuning of the constants as requiring some theoretical response, notably because the values of the constants appear arbitrary from a systematic point of view and exhibit no clear pattern (Donoghue 2007, Sect. 8). Moreover, two constants that profoundly shape the universe, the mass of the Higgs boson and the cosmological constant, are widely regarded as unnatural in that, based on theory-immanent grounds, one would expect their values to be of an entirely different order of magnitude. ${ }^{2}$ Thus there seems to be no compelling reason for the constants to have their values-in fact, other values would appear more natural — which is why many regard the fact that the universe is life-friendly as a profound mystery that calls for a theoretical response. ${ }^{3}$

One popular and influential such response to fine-tuning is to invoke divine creation (e.g. Swinburne 2004, 172-188). Another is to expect future developments in fundamental physics-for example an ambitious "theory of everything" as envisaged by Einstein (Schilpp 1949, 63) which would dictate the values of all parameters from first principles and thus undermine the request to explain their values. The multiverse idea, however, may offer a potentially attractive, non-theistic, less speculative alternative: if there is a sufficiently diverse multiverse, comprising many distinct universes with different values of the constants, it is only to be expected that there is at least one universe where they are right for life. As Carter's famous weak anthropic principle reminds us ${ }^{4}$, "our location ... is necessarily privileged to the extent of being compatible with our existence as observers" (Carter 1974, 293, emphasis due to Carter). Assuming that observers are generally living organisms, observers in the multiverse - if there are any-will unavoidably find the constants apparently right for life, even if the range of life-friendly values is strongly constrained. Along these lines the multiverse idea appears to be potentially able to account for why we exist despite the required fine-tuning.

This suggested inference from fine-tuning to a multiverse is an extremely remarkable instance of reasoning in physics: according to it, we are entitled to infer from the observation that our universe is life-friendly despite the required fine-tuning that there are (likely) many other universes (or distant regions of space-time) where the constants are different. It is no surprise, then, that some philosophers reject it as fallacious. Notably, it has been accused of committing what Hacking (1987) calls the inverse gambler's fallacy: inferring from an outcome that one regards as surprising or remarkable that there are likely many more events, most of them with much less surprising or remarkable outcomes.

Paradigmatically, the inverse gambler's fallacy is committed by someone who enters a casino, witnesses a remarkable outcome at the nearest table (a fivefold six in a toss of five dice, say), and infers that the overall number of tosses is (or has been) likely large. The inference is indeed fallacious under the assumption that the outcomes of the tosses are

\footnotetext{
1 For a review of six particularly dramatic instances of apparent fine-tuning, see Rees (2000), for a more complete recent book-length review see Lewis and Barnes (2016). Some apparently fine-tuned parameters describe the universe's boundary conditions (e.g. initial energy density and entropy) and may not strictly qualify as "constants". For the purposes of this paper one may regard them as included among the constants. ${ }^{2}$ See Williams (2015) for an enlightening discussion of naturalness aimed at philosophers.

${ }^{3}$ One can reject this view by arguing that we lack an independently motivated probability distribution over possible values of the constants that would make life-friendly constants surprising in an objective sense (McGrew et al. 2001). Somewhat relatedly, Callender (2004) objects against calls to account for apparently fine-tuned initial conditions. For the purposes of this paper I set aside this reaction.

4 See Barrow and Tipler (1986), Earman (1987), Leslie (1989), McMullin (1993), and Bostrom (2002) for discussions of this principle and for criticism some less plausible cousins.
} 
probabilistically independent because the outcomes neither influence each other nor have a common cause. ${ }^{5}$ According to White, the fine-tuning argument for the multiverse is guilty of this fallacy by "supposing that the existence of many other universes makes it more likely that this one-the only one that we have observed-will be life-permitting" (White 2000, 263). White's criticism, also known as the this universe objection, has been endorsed by other philosophers, notably Sober (2004), Draper et al. (2007), Titelbaum (unpublished) and Landsman (2016).

But not all philosophers agree with the this universe objection against the fine-tuning argument for the multiverse. ${ }^{6}$ Bradley (2009), for example, argues that the objection overlooks the observation selection effect which consists in the fact that, since we could not have existed in a life-hostile universe, our observations are biased towards finding constants that are right for life. According to him, if we take this effect into account, it becomes clear that the fine-tuning argument for the multiverse does not commit the inverse gambler's fallacy after all. However, Landsman (2016) has recently disputed the adequacy of Bradley's analogy, and consensus on whether the fine-tuning argument for the multiverse commits the inverse gambler's fallacy or not does not seem within reach.

The present paper considers two fine-tuning problems that are much more patently analogous to the problem of the fine-tuned constants than the urn and casino scenarios which have mostly been considered in the literature so far (reviewed in Sect. 2): the problem of our fine-tuned planet and the problem of our fine-tuned ancestors, introduced and discussed in Sects. 3 and 4, respectively. Reasoning that parallels the inference from fine-tuning to a multiverse seems prima facie adequate in these problems. But it turns out the inverse gambler's fallacy charge does not arise in them only due to the fact we have strong independent reasons to believe in the hypotheses that are analogous to the multiverse hypothesis in these problems. In the absence of such independent reasons it would be coherent, though perhaps not compelling, to raise the inverse gambler's fallacy charge against the inferences that parallel the inference from fine-tuning to a multiverse. In response to this diagnosis I suggest that we should seriously consider the possibility that established standards of rationality may just not allow us to decide whether the inference from fine-tuning to a multiverse commits the inverse gambler's fallacy or not. The paper concludes in Sect. 5 by sceptically assessing the prospects for obtaining compelling independent empirical evidence for concrete multiverse theories. Obtaining such evidence would be attractive inasmuch as it would make the inverse gambler's fallacy charge against the inference from fine-tuning to a multiverse obsolete.

\section{Urn Analogies}

Bradley (2009) illustrates the fine-tuning argument for the multiverse while defending it against the this universe objection in terms of an urn that contains either one or two balls, depending on the outcome of a fair coin toss. Balls come in two different sizes: large $(L)$ and small $(S)$. For each ball, an additional fair coin toss determines whether it is large or small, i.e. $P(L)=P(S)=1 / 2$ for each ball. A small hole is opened in the urn through

\footnotetext{
5 As Hacking (1987) introduces the fallacy, the fallacious conclusion is from one remarkable outcome in the present to the existence of many in the past. As highlighted by White (2000), though, there is still a fallacious inference if one infers the existence of simultaneous or future trials.

${ }^{6}$ For various lines of defence of the inference to many universes against Hacking's and White's considerations, see McGrath (1988), Leslie (1988), Bostrom (2002), Manson and Thrush (2003) and Juhl (2005).
} 
which a small ball is sampled if there is one, whereas a large ball would not fit through the hole. This makes the sampling procedure biased towards small balls and introduces an observation selection effect that must be accounted for in one's degrees of belief about how many balls there are in the urn. Indeed, if a small ball is sampled, this information is not neutral with respect to "one ball in the urn" versus "two balls in the urn", which it would be if the sampling procedure were not biased towards small balls. Sampling a small ball confirms "two balls in the urn" over "one ball in the urn" because, given "two balls", it was more likely that the urn would contain at least one small ball (which could be sampled) than given "one ball".

Bradley constructs an analogy between this example and the problem of the apparently fine-tuned constants by treating small balls as symbolizing life-friendly universes and large balls as symbolizing life-hostile ones. ${ }^{7}$ According to this analogy, just as we could not possibly sample a large ball from the urn, we could not have possibly found ourselves in a universe that is not life-friendly. And just as sampling a small ball from the urn confirms "two balls" over "one ball", Bradley claims, if we find ourselves in a universe where the constants are right for life, this supports "two universes" over "one universe". If " $N$ balls" (with large $N \gg 1$ ) is also a possibility, sampling a small ball confirms this even more strongly than "two balls". Analogously, " $N$ universes", corresponding to the multiverse hypothesis, is confirmed even more strongly than "two universes" when we find out that we exist despite the required fine-tuning.

Rational credences in this urn example are uncontroversial, but is the example itself really analogous to the problem of the fine-tuned constants? Klaas Landsman, one of those who believe that the fine-tuning argument for the multiverse commits the inverse gambler's fallacy, claims not and suggests that another urn example provides a much better analogy (Landsman 2016, Sect. 5). In Landsman's example, the competing hypotheses are not about how many balls there are in a single urn but about how many urns there are, where each urn contains only one ball, large or small, with $P(L)=P(S)=1 / 2$ for each urn independently. As before, only small balls can be sampled. Importantly, an observer's supervisions are confined to a single fixed urn. If she samples a small ball from that urn and concludes that there are likely more urns, she indeed commits the inverse gambler's fallacy. The selection bias in favour of small balls is irrelevant to the rational credences about the number of urns. No observation selection effect must be taken into account.

Landsman argues that this example can be recognized as relevantly analogous to the problem of the fine-tuned constants if we let urns, not balls, stand for universes. For a given urn, the size of its ball indicates whether the universe symbolized by the urn is life-friendly or not. According to Landsman, just as we have access to only a single fixed urn in the example, our observations are confined to a single fixed universe. If an observer samples a small ball from her urn, this corresponds to our finding that the constants in our universe are right for life. And just as sampling a small ball from one's urn is uninformative about whether there are more urns, finding the constants right for life in our universe is uninformative about whether there are more universes.

Which analogy is correct, Bradley's or Landsman's? It would be possible to answer this question with confidence only if it were clear whether it is rational for us to reason as we possibly could have lived in a different universe or not. Bradley's analogy assumes that it is rational to reason in that way: in his example, we can sample any small ball from the urn (if there is one), so the analogy presupposes that our existence is not tied to a specific universe

\footnotetext{
7 Inasmuch as one regards the probability for a universe to be life-friendly as very small, one may want to adjust the example so that the probability for a ball to be small is also very small: $P(S) \ll 1$.
} 
(corresponding to some specific ball), which may or may not be life-friendly. Landsman's analogy, in contrast, can only be adequate if it is not rational for us to reason as if we could have lived in a different universe: in his example, our attention is confined to a single urn with a single ball in it (large or small), which means that the analogy presupposes that our existence is tied to a specific universe (corresponding to a specific urn) that may or may not be life-friendly.

So, how should we reason? As one may have guessed (or feared), philosophers diametrically disagree on the answer to this question: White $(2000,269)$ argues that it would be irrational to reason as if we could have existed in a different universe, whereas Manson and Thrush (2003, 76f.), Juhl (2005, 345ff.) and Bradley $(2009,68)$ claim that there is no good reason for refraining from doing so.

Complementing his case against White, Bradley (2009, 68-70) presents an argument according to which even if, as White suggests, we should reason as if we could not have existed in a different universe, the existence of many universes would still make it more likely that the particular universe in which we could have existed-our own-indeed exists. White could object, however, that we should account for the life-friendliness of this universe, not its existence-and that its life-friendliness is not made any more likely by the existence of multiple other universes.

Perhaps there are further strong reasons-overlooked in the previous paragraphs-to believe that either Bradley's or Landsman's analogy is adequate whereas the other one is inadequate. In the absence of such reasons being brought forward, it seems natural to consider two further fine-tuning problems which are much more overtly analogous to the problem of the fine-tuned constants than the urn examples. Promisingly, there does not seem to be much controversy with respect to these problems about whether reasoning that parallels the fine-tuning argument for the multiverse commits the inverse gambler's fallacy.

\section{The Fine-Tuned Planet}

The first analogy - the problem of the fine-tuned planet-starts with the observation that life could not have appeared and evolved on Earth if Earth's size and mass, its distance from the sun, the size and distance of its neighbouring planets and the abundance of certain chemical elements on it had been significantly different. The parameters that describe these conditions on Earth appear fine-tuned for life in a similar way to how the constants of our universe appear fine-tuned for life. Earth's fine-tuning may seem less dramatic and impressive than the fine-tuning of the constants, ${ }^{8}$ but there does not seem to be any principled difference between the two: inasmuch as the life-friendliness of the universe is surprising in view of the required fine-tuning of the constants, the life-friendliness of Earth is at least prima facie surprising in view of the required fine-tuning of the factors mentioned. ${ }^{9}$

\footnotetext{
${ }^{8}$ See Ward and Brownlee (2000) for a defence of the view that Earth's apparent fine-tuning for life is dramatic. More recent research suggests, however, that the proportion of life-friendly planets may actually be significantly higher than previously assumed (Loeb et al. 2016). The problem of the fine-tuned planet is discussed as a candidate analogy to the problem of the fine-tuned constants in Manson and Thrush (2003, 73) and Greene (2011, 169f.).

9 A potentially relevant difference is that the fine-tuning of the constants is a fine-tuning within the laws of nature (since the constants appear in the laws), whereas Earth's fine-tuning is a merely local affair. One could highlight this difference in an attack against the fine-tuning argument, arguing that it does not make sense to demand an explanation for the fine-tuned constants since their values could not have been different
} 
Unlike the apparent fine-tuning of the constants, Earth's apparent fine-tuning for life is not widely perceived as a profound puzzle. There does not seem to be any research activity that is directed, for example, at constructing a theory of planet formation according to which planets - or, more realistically, planets of a certain type, exemplified by Earth — are in general life-friendly, as a consequence of the physical laws, which would make Earth's life-friendliness entirely expectable. The most straightforward reason as to why we do not feel that such a theory would be helpful is that, when assessing the evidential relevance of Earth's apparent fine-tuning, we seem to intuitively take into account an observation selection effect similar to the one invoked by proponents of the fine-tuning argument for the multiverse: it has been known for a long time that there are other planets beside Earth-enormously many, according to relatively recent discoveries of extrasolar planets; and, given the enormous size of our universe, it is only to be expected that at least some of the many planets in our universe are life-friendly; finally, that we live on one of the (comparatively rare) life-friendly ones is unsurprising since we could not have possibly found ourselves on any of the others (nor anywhere else in life-hostile interplanetary space, for that matter).

According to this line of thought, Earth's life-friendliness can be elegantly explained by appeal to the long suspected, now established, existence of an enormous number of extrasolar planets, many of them not life-friendly, in combination with an observation selection effect. Call this perspective on Earth's apparent fine-tuning the many planets response to planetary fine-tuning. According to it, if we lacked any observational evidence for the existence of other planets besides Earth, it would be rational for us to infer that there are likely many other planets besides Earth, many of them inhospitable to life. The many planets response is closely analogous to the many universes response to apparent cosmic fine-tuning, as the rows "Our universe" and "Our planet (Earth)" in Table 1 indicate in a side-by-side exposition. (The fine-tuning problem "Our ancestors", outlined in the third row, is discussed in the following section.)

In analogy to the this universe objection against the fine-tuning argument for the multiverse one can set up a this planet objection against the many planets response to planetary fine-tuning. The this planet objection contends that the existence of many other planets does nothing to explain why Earth is life-friendly. In analogy to the this universe response, it insists that we should not reason as if we could have existed on a different planet. According to it, if there were no independent evidence for many other planets, inferring their existence from Earth's fine-tuning for life would mean comitting the inverse gambler's fallacy. Manson and Thrush briefly consider the possibility that one might endorse this objection and dismiss it:

[A]ccounts that appeal to the vast number of planets in our universe (and hence the vast number of chances for conditions to be just right) surely are not to be faulted for failing to explain why this planet is the fit one. Clearly the "This Planet" objection (TP) is no good[...]. (Manson and Thrush 2003, 73)

Footnote 9 continued

as a matter of physical necessity. (Colyvan et al. (2005) explore a criticism of fine-tuning arguments, both for God and for the multiverse, along these lines.) This attack, however, whether successful or not, is unrelated to the this universe objection and therefore ignored in what follows. 
Table 1 Three types of apparent fine-tuning

\begin{tabular}{|c|c|c|}
\hline & Type of apparent fine-tuning & Many ... response \\
\hline Our universe & $\begin{array}{l}\text { Values of the constants and cosmic } \\
\text { boundary conditions right for life } \\
\text { (cosmic fine-tuning) }\end{array}$ & $\begin{array}{l}\text { There are multiple universes, most with } \\
\text { the wrong constants and wrong } \\
\text { boundary conditions for life, a few, } \\
\text { including ours, with the right ones. As } \\
\text { observers, we had to find ourselves in } \\
\text { a universe with the right constants }\end{array}$ \\
\hline Our planet (Earth) & $\begin{array}{l}\text { Earth's size and age, distance from } \\
\text { central star, abundance of chemical } \\
\text { elements etc. right for life (planetary } \\
\text { fine-tuning) }\end{array}$ & $\begin{array}{l}\text { There are multiple planets, most with the } \\
\text { wrong size and age, distance from } \\
\text { central star, abundance of chemical } \\
\text { elements etc., a few, including Earth, } \\
\text { with the right ones. As observers, we } \\
\text { had to find ourselves on a planet with } \\
\text { the right conditions }\end{array}$ \\
\hline $\begin{array}{l}\text { Our ancestors (over } \\
\text { the last } 500 \\
\text { hundred million } \\
\text { years) }\end{array}$ & $\begin{array}{l}\text { Highly adapted to perennially changing } \\
\text { environmental conditions, competitive } \\
\text { in continuous struggle for survival and } \\
\text { reproductive opportunities, capable to } \\
\text { raise infants etc. (organismic fine- } \\
\text { tuning) }\end{array}$ & $\begin{array}{l}\text { There were multiple siblings (and } \\
\text { cousins etc.) of our ancestors, many of } \\
\text { them less well adapted to their } \\
\text { environments than our ancestors, less } \\
\text { competitive in continuous struggle for } \\
\text { survival and reproductive } \\
\text { opportunities, less capable to raise } \\
\text { infants etc. Evidently, our ancestors } \\
\text { were among the reproductively } \\
\text { successful organism. It is only to be } \\
\text { expected that, as such, they were } \\
\text { among the particularly well adapted } \\
\text { ones }\end{array}$ \\
\hline
\end{tabular}

Our ancestors (over he last 500 hundred million years) environmental conditions, competitive reproductive opportunities, capable to raise infants etc. (organismic finetuning)
There are multiple universes, most with observers, we had to find ourselves in

There are multiple planets, most with the wrong size and age, distance from central star, abundance of chemical with the right ones. As observers, we had to find ourselves on a planet with

There were multiple siblings (and many of mess well adapted to their competitive in continuous struggle for survival and reproductive pportunities, less capable to raise were among the reproductively successful organism. It is only to be expected that, as such, they were ones

However, there is a simple move that at least in principle allows its proponents to defend the this planet objection: they can claim that Earth's life-friendliness may just be a primitive lucky coincidence which we have to accept as such. ${ }^{10}$ According to this lucky coincidence response to Earth's apparent fine-tuning in combination with the this planet objection, the existence of many other planets does not make it more likely that our planet is life-friendly and, so, contributes nothing to the explanation of that finding. And, according to this line of thought, in the absence of independent observational evidence for other planets the inference from Earth's fine-tuning to their existence would commit the inverse gambler's fallacy, just like the inference from fine-tuned constants to a multiverse.

Interestingly, in our actual epistemic situation, where we do have independent empirical evidence for many other planets besides Earth, it does not matter much for scientific practice whether one accepts the many planets or the lucky coincidence response to Earth's fine-tuning: proponents of both responses can agree that attempts to explain Earth's lifefriendliness by appeal to the laws of physics or by appeal to some divine designer are neither needed nor promising any more. From the perspective of the many planets response, the existence of many other planets besides Earth already provides a satisfactory explanation of why Earth is life-friendly. From the perspective of the lucky coincidence response the existence of many other, mostly life-hostile, planets, is also relevant, though

${ }^{10}$ This reaction is not new, at least not in spirit. With respect to the problem of the fine-tuned constants a stance along these lines is defended by Gould (1983) and Carlson and Olsson (1998). 
in a very different way, namely, in that it shows that planets in general are just not lifefriendly, which in turn indicates that no law-based or designer-based explanation of why Earth, qua being a planet, had to be life-friendly will succeed.

\section{The Fine-Tuned Ancestors}

Consider our ancestors over the last 500 million years and picture their course of evolution across generations. ${ }^{11}$ Now, relying on your awareness of the dangers that threaten animals in the wild and the challenges to their successful reproduction, estimate what the odds were for the members of such a large class of organisms to survive (without exception) into reproductive age, reproduce, and successfully raise at least some of their young: no doubt exceedingly low! Our ancestors must have been extremely well adapted in order to overcome all those permanent threats to their survival and reproductive success.

A key component of the correct response to this apparent organismic fine-tuning of our ancestors (see the third row in Table 1) is the standard-and no doubt appropriateDarwinian account of natural selection as the main mechanism of why all organisms, not only our ancestors, were and are apparently fine-tuned in the sense of being highly adapted (while other factors beside natural selection, notably genetic drift, mutation, and migration, also play crucial roles in evolution). At any stage of evolution, organisms that are better adapted generally have better chances to survive and reproduce. As a result, organisms continue being adapted over generations even when selection pressures vary over time.

Note that in this appeal to natural selection to account for our ancestors' apparent finetuning we have to include their same-species companions in the picture, e.g. their siblings and cousins, notably those who either did not survive into reproductive age or did so but failed to reproduce (or became the ancestors only of non-human organisms). Natural selection requires almost permanent "overproduction" of organisms in order to not lead into terminal population decline, so in that sense this reply to fine-tuning invokes "many organisms".

Clearly though, despite its appeal to "many organisms" in this sense, this response to our ancestors' apparent fine-tuning differs fundamentally from many planets and many universes: while the latter are centred around appeal to an observation selection effect, the response to our ancestors' apparent fine-tuning just sketched is based on an appeal to natural selection. However, if we try to account for our ancestors' full apparent fine-tuning, it turns out that the appeal to natural selection does not suffice: we must either add an appeal to an observation selection effect or invoke sheer luck. ${ }^{12}$

This can be seen as follows: in view of the theory of natural selection itself it is reasonable to expect that those organisms that survive into reproductive age and actually reproduce, when compared to their same-species contemporaries, are in general particularly well adapted to the dominant selection pressures of the day. Their "degree of apparent fine-tuning", inasmuch as well-defined, is typically above average. As a consequence of their reproductive success, their phenotypic traits correlate more strongly with the phenotypic traits of next-generation organisms than those of their contemporaries with less

\footnotetext{
11 The biological layperson interested in performing this exercise in imagination may profit from consulting (Dawkins 2004) for a lively account of our extended evolutionary history, which focuses in particular on what is known about our common ancestors with other extant species.

12 Interestingly, Smolin (2007) offers a version of the many universes response to cosmic fine-tuning that applies natural selection at the cosmic level. Assessing this speculative proposal is beyond the scope of this paper, though.
} 
reproductive success. Thus, at each evolutionary stage, the successfully reproducing organisms, unlike their contemporaries, seem to correctly "anticipate" the subsequent turns of evolution.

Evidently, all our ancestors were so lucky to survive into reproductive age and reproduce, so most of them were probably particularly well adapted and correctly "anticipated" subsequent turns of evolution. But why were they so lucky or, equivalently, why were we so lucky that none of our ancestors failed to survive into reproductive age and to reproduce?

There are two coherent ways to respond to this question. The first, which in analogy to many universes and many planets may be called many organisms, invokes an observation selection effect: our ancestors are not "randomly chosen" organisms in the evolutionary history of our species. We focused on them by using a criterion-being our ancestorswhich entails survival into reproductive age and successful reproduction. It is only to be expected that organisms that conform to this criterion are on average as adapted ("finetuned") as reproductively successful organisms usually are.

But there is a second coherent way to respond, namely to refuse giving any explanation of why our ancestors were so particularly well adapted beyond citing sheer luck. Evidently, this reaction parallels the lucky coincidence response to Earth's apparent fine-tuning encountered in the previous section. The latter reaction seems coherent as well.

The ideological gulf between both responses may seem deep. Notably, those who opt for the lucky coincidence response may claim that those who adopt many organisms commit the inverse gambler's fallacy. On the level of scientific practice, however, there seem to be few significant differences, which is due to the fact that there are very strong independent reasons to believe that our ancestors had many same-species contemporaries many of whom were less well adapted to the dominant environmental selection pressures of the day than our ancestors were. Given this shared belief, all parties agree that it is unpromising to try to explain why precisely our ancestors-rather than some of their reproductively less lucky contemporaries, say-were so well adapted that their chances to survive and reproduce were comparatively high. A single scientific individual may switch forth and back between both perspectives-"lucky coincidence" versus "observation selection effect" - without displaying any irritating or incoherent behaviour in practice. To conclude, because we have strong independent reasons to believe that our ancestors had multiple siblings who were less lucky, reflecting on them, while illuminating and instructive in itself, has not given us any novel reason to believe that we can determinately assess whether reasoning that has the same form as the fine-tuning argument for the multiverse is fallacious or not.

\section{Back to the Multiverse}

Apart from the fact that natural selection plays a crucial role in the problem of our finetuned ancestors, the two problems discussed in the previous sections still seem relevantly analogous to the problem of the fine-tuned constants. The absence of debate concerning what we can rationally infer from these instances of fine-tuning seems to be a consequence of the fact that we have independent empirical evidence for other planets and for multiple sibling organisms of our ancestors. That absence of debate does therefore not suggest that the inference from fine-tuning to the respective many-response is non-fallacious. Indeed, if we did not have such independent evidence, one could reasonably level the inverse gambler's fallacy charge against the inference from the fine-tuned planet to multiple 
planets and against the inference from our fine-tuned ancestors to multiple sibling organisms. As a consequence, considerations on the fine-tuned planet and the fine-tuned ancestors unfortunately do not help us to a verdict on whether the inference from our universe's fine-tuning for life to many other universes is fallacious or not. Whereas arguments based on urn and casino scenarios suffer from the fact that one can doubt whether those scenarios are really relevantly analogous, the fine-tuned planet and finetuned ancestors problems are ultimately of little help because, were it not for the existence of independent evidence for many planets and many organisms, the dialectical situation with respect to them would exactly parallel the dialectical situation with respect to the finetuned constants. These difficulties may be principled: perhaps the problem of the fine-tuned constants is just too different from any problem that has an agree-upon solution. We should take the possibility seriously that the question of whether the inference from fine-tuning for life to multiple universes commits the inverse gambler's fallacy simply has no determinate answer, at least not in the light of established standards of rationality.

What would our epistemic situation be like if we had convincing independent empirical evidence for many other universes with different constants (or convincing independent evidence that the constants differ across space-time in our own universe)? We would then be in a situation with respect to the fine-tuning of the constants that is similar to our actual situation with respect to the Earth's and our ancestors' fine-tuning: we could either regard the life-friendliness of the constants as elegantly explained by the (independently established) existence of the other universes in combination with an observation selection effect; or we could regard the project of explaining why the constants have the values that they have where we are as obsolete because there would evidently be no principled reasons as why they are what they are where we are. Notably, if we had independent evidence for other universes with different constants, the appeal to a divine designer to explain why our own universe is life-friendly would lose its appeal. As conceded by White, "while we might suppose that a designer would create some intelligent life somewhere, there is little reason to suppose it would be here rather than in one of the many [hypothesized] other universes" (White 2000, 273f.).

Can we hope to obtain independent empirical support for the existence of other universes if such universes exist? One can think of multiverse theories that are indeed testable in a relatively straightforward manner, namely, those according to which causal traces of the other universes can ultimately be detected in our universe after all. In the nowadays most prominent multiverse scenario-the so-called landscape multiverse (Susskind 2005) - this could be the case in that there might be "bubble collisions" between distinct universes which would leave detectable traces in the colliding universes. Identifying those traces and making the right inferences about their origins is an intricate challenge (Aguirre and Johnson 2011; Salem 2012). If it can be accomplished, obtaining empirical evidence about other universes - inasmuch as those distinct "bubbles" then deserve to be called distinct "universes" at all—is not in principle different from obtaining empirical evidence about, say, distant galaxies or about the very early universe and does not pose any particular epistemological challenges beyond those already present in ordinary (single-universe) cosmology.

But what if the other universes in the multiverse are not in causal contact with our own? At least in principle, even multiverse theories with this consequence can be tested like other physical theories, namely, as Sean Carroll calls it, by means of "abduction, Bayesian inference, and empirical success" (Carroll (2018), abstract), (see also Stoeger (2007) for considerations about obtaining independent evidence for multiverse theories). What Carroll has in mind may work best in cases where, as considered by Greene (2011, Chapter 7), 
a multiverse theory $T$ entails that the value of some parameter $\lambda$ is very close to some specific value $\lambda_{0}$ in all subuniverses. In that case, if we find the value of $\lambda$ to be $\lambda_{0}$ in our universe and rival theories do not entail this finding, we may regard it as evidence in favour of that multiverse theory $T$.

Unfortunately, there is no reason to expect that this-from the point of view of theory assessment - fortunate scenario will be realized for actual candidate multiverse theories like the landscape multiverse. And for theories that entail the existence of universes with very different values of a large number of parameters $\lambda$, it is at least prima facie completely unclear how we should assess the evidential impact of our observations with respect to them.

The most popular and important move that proponents of concrete multiverse theories such as the landscape multiverse have suggested in response to these difficulties is to test those theories by combining them with the assumption that we are randomly selected from some suitably chosen reference class of multiverse inhabitants. ${ }^{13}$ According to this assumption, we should treat the multiverse theories like the landscape multiverse scenario as predicting that our measurement results will be typical among those obtained by observers in the multiverses that exist according to those theories.

However, typicality assumptions are controversial (Hartle and Srednicki 2007), they require the difficult choice of an appropriate observer reference class (Friederich 2017), and they are not straightforwardly applicable if observer numbers are infinite, as seems to be the case for the landscape multiverse. ${ }^{14}$ To conclude, even if some concrete multiverse theory is correct, obtaining compelling independent empirical evidence for it will be very difficult. But obtaining such evidence may be necessary to confidently assess the rational significance of the observation that the constants seem fine-tuned for life.

Open Access This article is distributed under the terms of the Creative Commons Attribution 4.0 International License (http://creativecommons.org/licenses/by/4.0/), which permits unrestricted use, distribution, and reproduction in any medium, provided you give appropriate credit to the original author(s) and the source, provide a link to the Creative Commons license, and indicate if changes were made.

\section{References}

Aguirre, A. (2007). Making predictions in a multiverse: Conundrums, dangers, coincidences. In B. Carr (Ed.), Universe or Multiverse? (pp. 367-386). Cambridge: Cambridge University Press.

\footnotetext{
13 Bostrom dubs this the self-sampling assumption (SSA). It is a variant of similar ideas developed and refined by Carter (1974), Gott (1993), Vilenkin (1995), Elga (2004), Aguirre (2007) and Barnes (2017).

In addition to the SSA, Bostrom considers the self-indication assumption (SIA). The SIA applies in contexts where we consider the rational impact of empirical evidence with respect to competing scientific hypotheses, and it states that the rational prior probabilities to assign to those hypotheses are proportional to the numbers of observers that exist according to them. In other words, the SIA advises us to systematically prefer hypotheses that entail the existence of more observers over hypotheses that entail the existence of fewer observers. The SIA is not widely accepted (see Bostrom 2002, 124, for a very critical assessment by Bostrom himself), and even if it were widely accepted, this would not contribute to resolving the singleuniverse versus multiverse debate: that debate concerns whether the constants (or laws) are uniform or variable, not whether the total number of observers is comparatively small or large.

14 The difficulty that arises in infinite settings with infinite observer numbers is known as the "measure problem" of cosmology. See Schellekens (2013) for a review of string theory that includes a pedagogical introduction to it, (Smeenk 2014) for a philosopher's sceptical assessment of its resolvability, and (Arntzenius and Dorr 2017) for a more optimistic perspective.
} 
Aguirre, A., \& Johnson, M. C. (2011). A status report on the observability of cosmic bubble collisions. Reports on Progress in Physics, 74, 074901.

Arntzenius, F., \& Dorr, C. (2017). Self-locating priors and cosmological measures. In K. Chamcham, J. Barrow, S. Saunders, \& J. Silk (Eds.), The philosophy of cosmology (pp. 396-428). Cambridge: Cambridge University Press.

Barnes, L. A. (2017). Testing the multiverse: Bayes, fine-tuning and typicality. In K. Chamcham, J. Barrow, S. Saunders, \& J. Silk (Eds.), The philosophy of cosmology (pp. 447-466). Cambridge: Cambridge University Press.

Barrow, J. D., \& Tipler, F. J. (1986). The anthropic cosmological principle. Oxford: Oxford University Press.

Bostrom, N. (2002). Anthropic bias: Observation selection effects in science and philosophy. New York: Routledge.

Bradley, D. J. (2009). Multiple universes and observation selection effects. American Philosophical Quarterly, 46, 61-72.

Callender, C. (2004). Measures, explanations and the past: Should 'special' initial conditions be explained? British Journal for the Philosophy of Science, 55, 195-217.

Carlson, E., \& Olsson, E. J. (1998). Is our existence in need of further explanation? Inquiry, 41, 255-275.

Carroll, S. M. (2018). Beyond falsifiability: Normal science in a multiverse. arxiv:1801.05016. Accessed 12 June 2018.

Carter, B. (1974). Large number coincidences and the anthropic principle in cosmology. In M. S. Longair (Ed.), Confrontation of cosmological theory with astronomical data (pp. 291-298). Dordrecht: Reidel.

Colyvan, M., Garfield, J. L., \& Priest, G. (2005). Problems with the argument from fine-tuning. Synthese, $145,325-338$.

Dawkins, R. (2004). The Ancestor's Tale: A pilgrimage to the dawn of evolution. New York: Houghton Mifflin.

Donoghue, J. F. (2007). The fine-tuning problems of particle physics and anthropic mechanisms. In B. Carr (Ed.), Universe or Multiverse? (pp. 231-246). Cambridge: Cambridge University Press.

Draper, K., Draper, P., \& Pust, J. (2007). Probabilistic arguments for multiple universes. Pacific Philosophical Quarterly, 88, 288-307.

Earman, J. (1987). The SAP also rises: A critical examination of the anthropic principle. Philosophical Quarterly, 24, 307-317.

Elga, A. (2004). Defeating Dr. Evil with self-locating belief. Philosophy and Phenomenological Research, $69,383-396$.

Friederich, S. (2017). Resolving the observer reference class problem in cosmology. Physical Review D, 95 , 123520.

Gott, R. (1993). Implications of the Copernican principle for our future prospects. Nature, 363, 315-319.

Gould, S. J. (1983). Mind and supermind. Natural History, 92, 34-38.

Greene, B. (2011). The hidden reality. New York: Vintage.

Hacking, I. (1987). The inverse gambler's fallacy: The argument from design. The anthropic principle applied to Wheeler Universes. Mind, 96, 331-340.

Hartle, J., \& Srednicki, M. (2007). Are we typical? Physical Review D, 75, 123523.

Juhl, C. (2005). Fine-tuning, many worlds, and the 'inverse gambler's fallacy'. Nous, 39, 337-347.

Landsman, K. (2016). The fine tuning argument. In K. Landsman, \& E. van Wolde (Eds.), The challenge of chance (pp. 111-128). Heidelberg: Springer.

Leslie, J. (1988). No inverse gambler's fallacy in cosmology. Mind, 97, 269-272.

Leslie, J. (1989). Universes. London: Routledge.

Lewis, G. J., \& Barnes, L. A. (2016). Fortunate universe: Life in a finely tuned cosmos. Cambridge: Cambridge University Press.

Loeb, A., Batista, R. A., \& Sloan, D. (2016). Relative likelihood for life as a function of cosmic time. Journal of Cosmology and Astroparticle Physics, 08, 40.

Manson, N. A., \& Thrush, M. (2003). Fine-tuning, multiple universes, and the 'this universe' objection. Pacific Philosophical Quarterly, 84, 67-83.

McGrath, P. J. (1988). The inverse gambler's fallacy and cosmology-A reply to Hacking. Mind, 97, 331-340.

McGrew, T., McGrew, L., \& Vestrup, E. (2001). Probabilities and the fine-tuning argument: A sceptical view. Mind, 110, 1027-1038.

McMullin, E. (1993). Indifference principle and anthropic principle in cosmology. Studies in History and Philosophy of Science, 24, 359-389.

Rees, M. (2000). Just six numbers: The deep forces that shape the universe. New York: Basic Books. 
Salem, M. P. (2012). Bubble collisions and measures of the multiverse. Journal of Cosmology and Astroparticle Physics, 2012(01), 021.

Schellekens, A. N. (2013). Life at the interface of particle physics and string theory. Reviews of Modern Physics, 85, 1491.

Schilpp, P. (Ed.). (1949). Albert Einstein: Philosopher-Scientist. Peru, IL: Open Court Press.

Smeenk, C. (2014). Predictability crisis in early universe cosmology. Studies in History and Philosophy of Modern Physics, 46, 122-133.

Smolin, L. (2007). Scientific alternatives to the anthropic principle. In B. Carr (Ed.), Universe or Multiverse (pp. 323-366). Cambridge: Cambridge University Press.

Sober, E. (2004). The design argument. In W. E. Mann (Ed.), The Blackwell guide to the philosophy of religion (pp. 117-147). Oxford: Blackwell Publishers.

Stoeger, W. R. (2007). Are anthropic arguments, involving multiverses and beyond, legitimate? In B. Carr (Ed.), Universe or Multiverse? (pp. 445-458). Cambridge: Cambridge University Press.

Susskind, L. (2005). The cosmic landscape: String theory and the illusion of intelligent design. New York: Back Bay Books.

Swinburne, R. (2004). The existence of god (2nd ed.). Oxford: Clarendon.

Titelbaum, M. G. (Unpublished). De re evidence and the anthropic argument for the multiverse. Available online at https://sites.google.com/site/michaeltitelbaum/research.

Vilenkin, A. (1995). Predictions from quantum cosmology. Physical Review Letters, 74, 846-849.

Ward, P., \& Brownlee, D. E. (2000). Rare earth: Why complex life is uncommon in the universe. New York: Copernicus.

White, R. (2000). Fine-tuning and multiple universes. Nous, 34, 260-267.

Williams, P. (2015). Naturalness, the autonomy of scales, and the $125 \mathrm{GeV}$ Higgs. Studies in History and Philosophy of Modern Physics, 51, 82-96. 